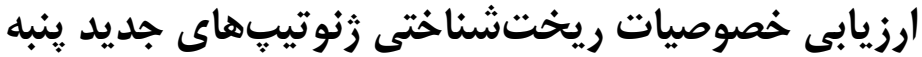 (Gossypium hirsutum L.) \\ آيدين حميدى '، كمال قاسمى بزدى 'و ياسر جعفرى
}

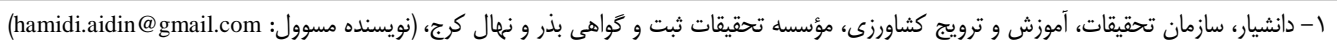

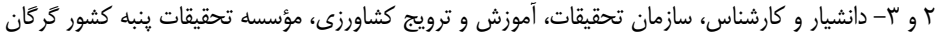
تاريخ هذيرش: ت q تاريخ دريافت:

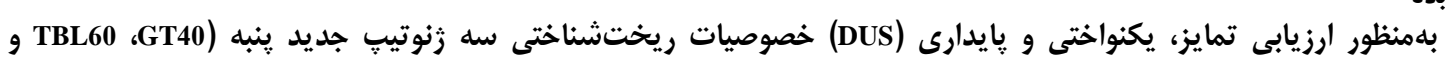

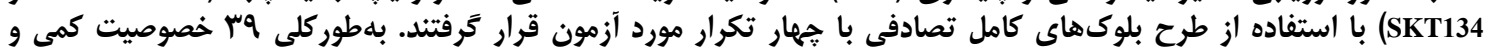

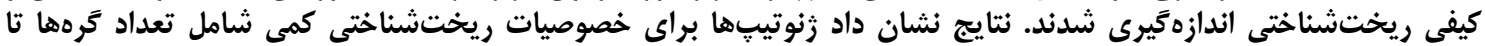

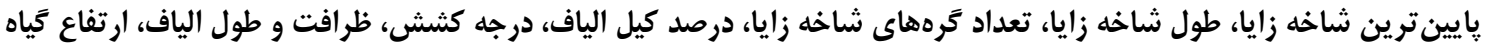

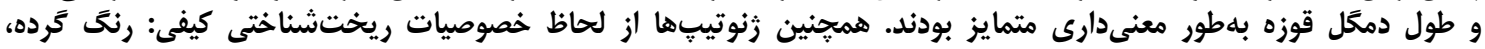

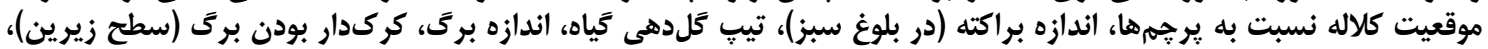

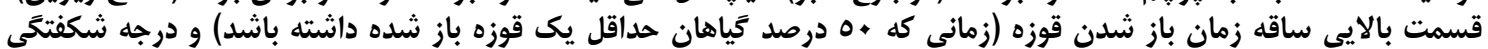

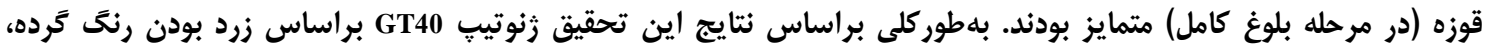

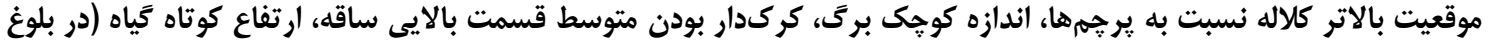

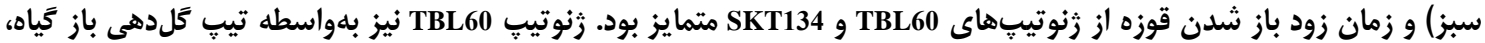

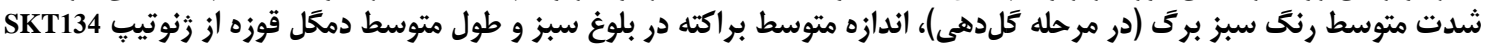

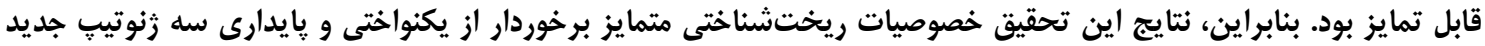

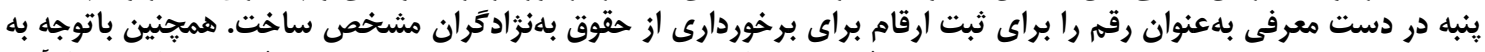

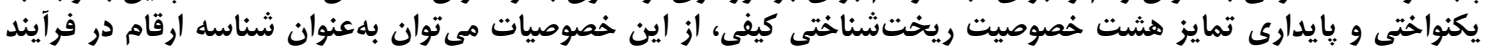

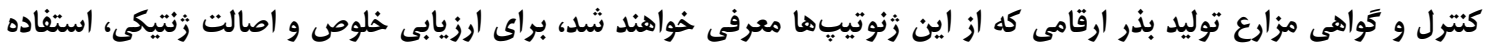

نمود.

وازههاى كليدى: خصوصيات ريختشناختى، ينبه، ثبت رقم، تمايز، يكنواختى، پايدارى

مهمترين خصوصيات ارزيابى تمايز رقم هستند (Tسّ). اتحاديسه

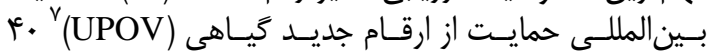

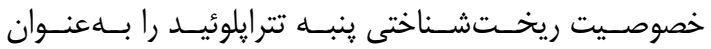

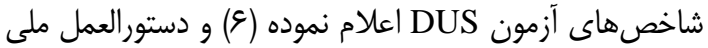

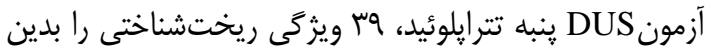

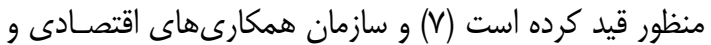

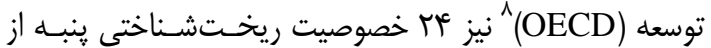

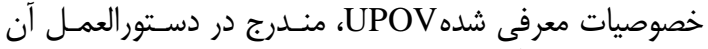

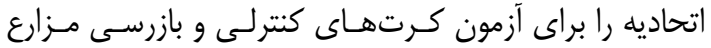

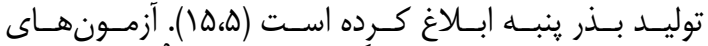

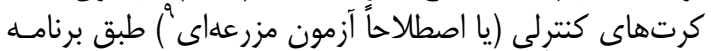

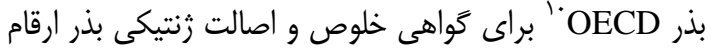

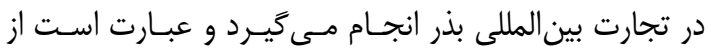

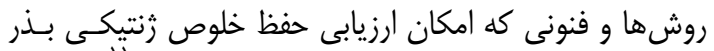

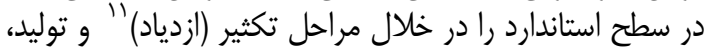

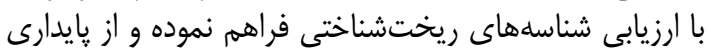

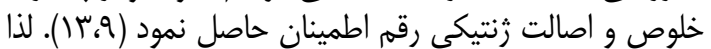

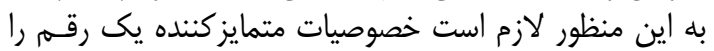

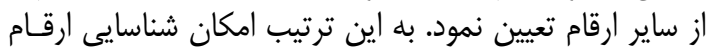

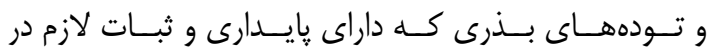

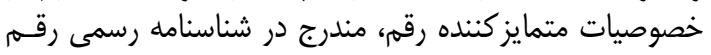

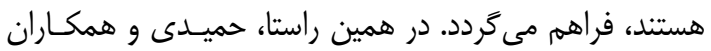

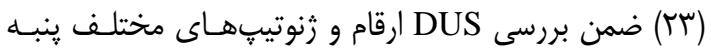

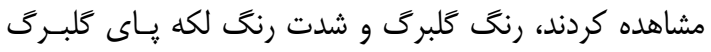

مقدمله

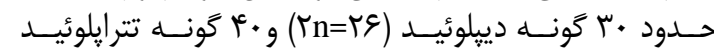
(rn=Dr)

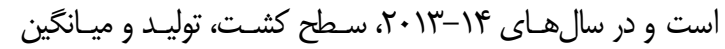

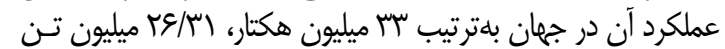

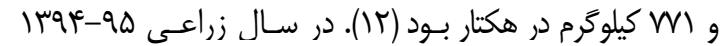

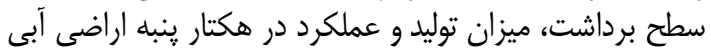

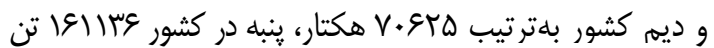

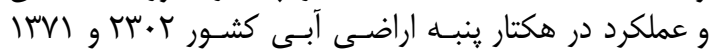

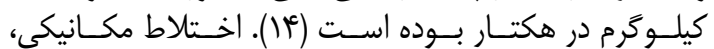

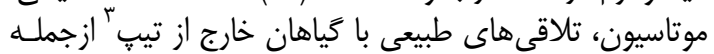

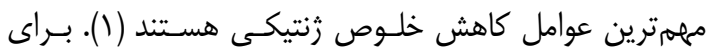

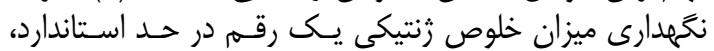

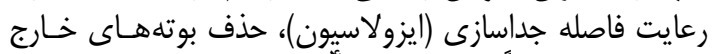

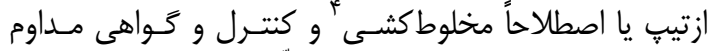

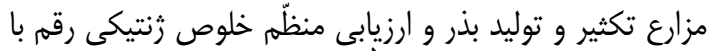

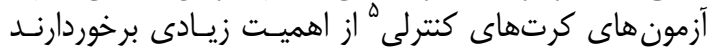

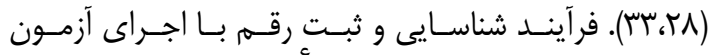

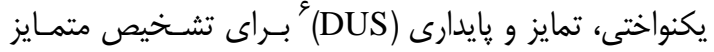

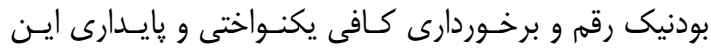

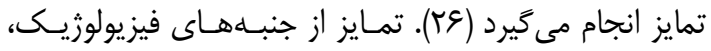

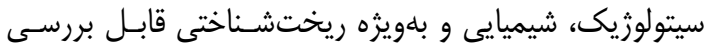

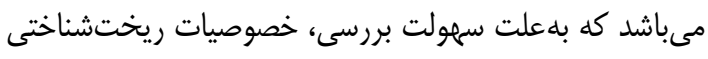

1-Gossypium spp. 2- Malvaceae 3- Off- type

6- Distinctness, uniformity and stability (DUS) test $\quad$ 7- International Union for the Protection of New Varieties of Plants (UPOV) 8- Organization for Economic Co-operation and Development (OECD) 


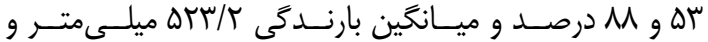

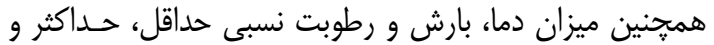

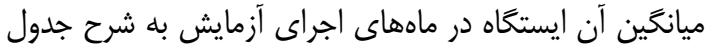

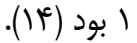
بلمنظور ارزيابى خصوصيات ريختشناختى كمى و كيفى الري

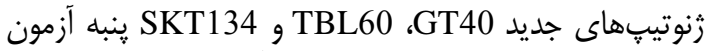

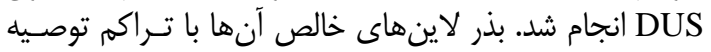

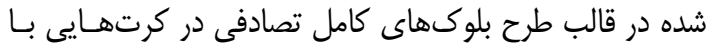

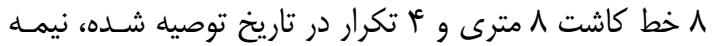

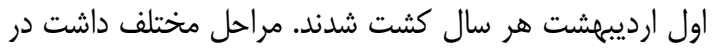

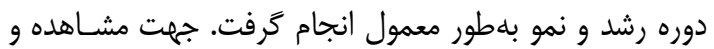

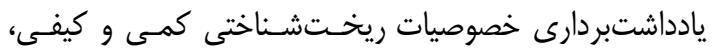

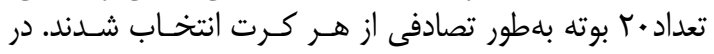

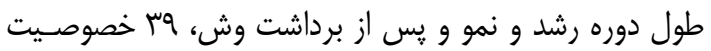

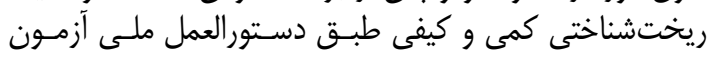
هUS

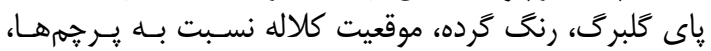

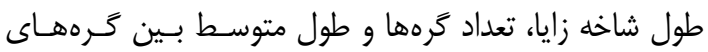

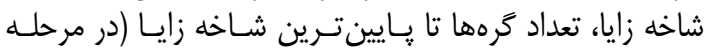

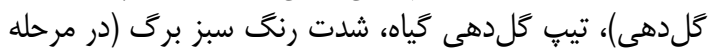

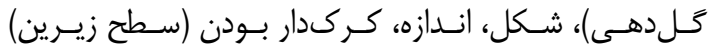

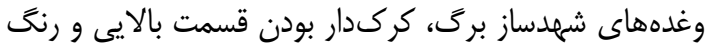

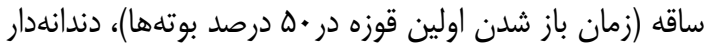

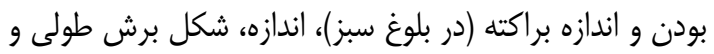

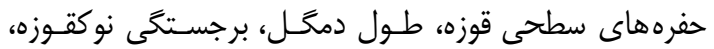

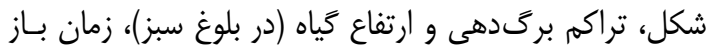

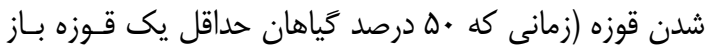

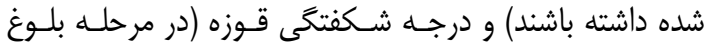

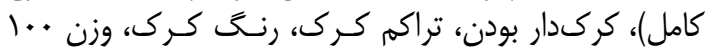

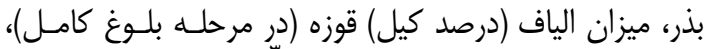

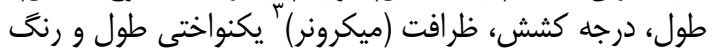

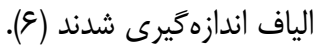

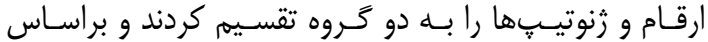

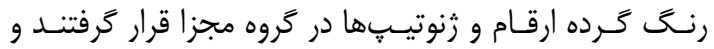

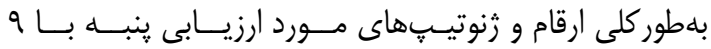

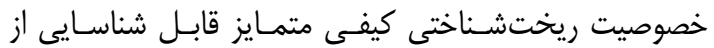
يكديگر بودند. هشت رقم ورامين، ساحل، بختخان و مهر كلستان، ارمغان

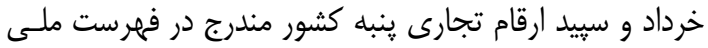

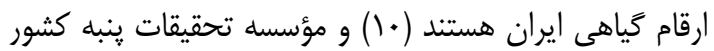

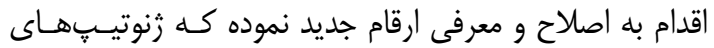

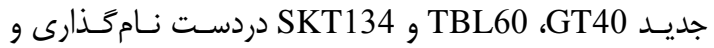
معرفى مىباشند. لذا، باتوجه به اهميت شناسـايى خصوصـيـ

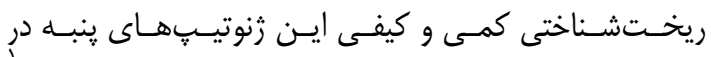

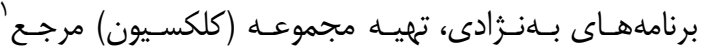

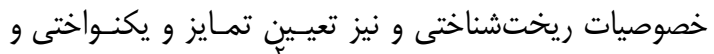

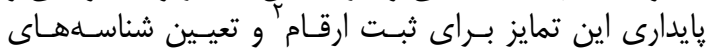

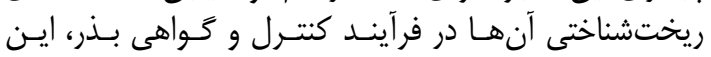

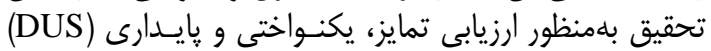

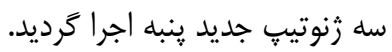

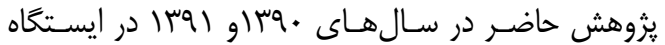

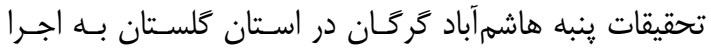

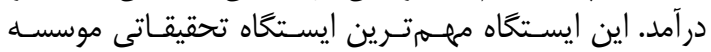

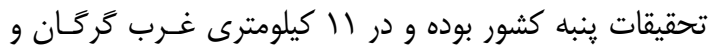

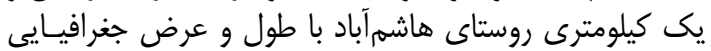

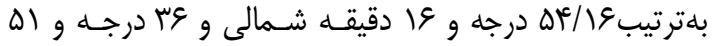

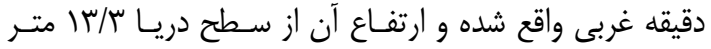

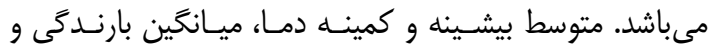

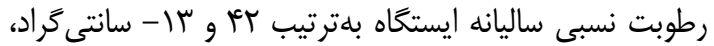

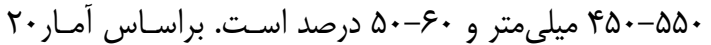

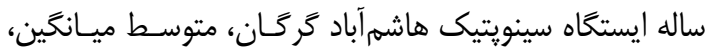

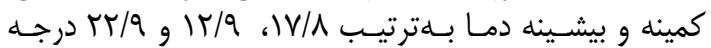

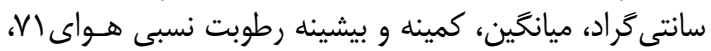

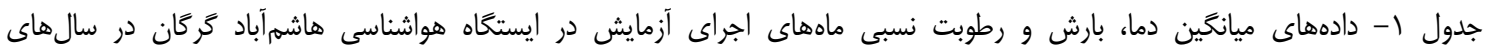

(1)

Table 1. Average temperature, precipitation and relative humidity date of Hashem Abad Gorgan meteorology station

\begin{tabular}{|c|c|c|c|c|}
\hline ميانگين رطوبت نسبى & ميانخين بارش (ميلىمتر) & ميانكَين دما (درجهسانتى كراد) & ماه & سال \\
\hline $\mathrm{VA}$ & $1 / \mu r$. & $1 / / 9$. & ارديبهشت & \\
\hline سو & $\Delta / F r$. & $r \omega / v$. & خرداد & \\
\hline Gr & $r / \cdot \Delta)$ & rN/q. & تير & וrq. \\
\hline gr & r & $\Gamma \cdot / \mu$. & مرداد & \\
\hline 99 & $r / .+q q$ & $r \Delta / r$. & شهريور & \\
\hline v. & V/r/ & $r / /$. & مهر & \\
\hline س & $r / v \cdot r$ & $r \Delta / \cdot r$ & & ميانكين \\
\hline 90 & س. & $r) / q$. & ارديبهشت & \\
\hline$\Delta \Lambda$ & $r / .1 f$ & re/G. & خرداد & \\
\hline 99 & q/ו & $T V / T$. & تير & $\mid 1 \% q$ \\
\hline $9 \Delta$ & $\mu / \phi_{. .}$ & $r T / l$. & مرداد & \\
\hline v. & $\Gamma / \cdot \Delta$. & $r E / T$. & شهريور & \\
\hline Vr & $N / \cdot \cdot 1$ & $r \cdot / \Lambda$. & مهر & \\
\hline$s q / 0$. & ه/द्रq & $r \Delta / \wedge \Delta$ & & \\
\hline
\end{tabular}




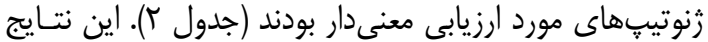

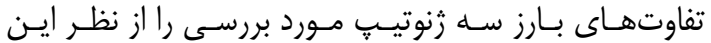

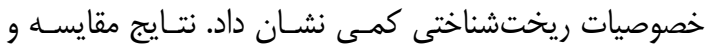

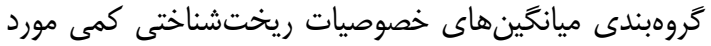

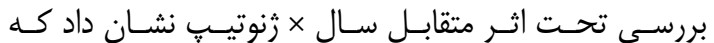

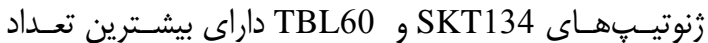

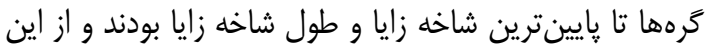

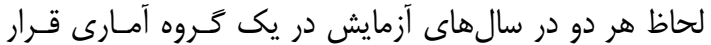

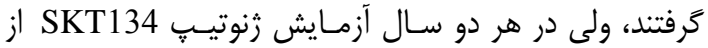

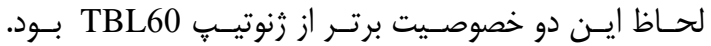

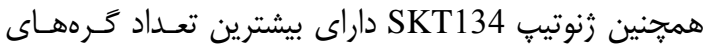

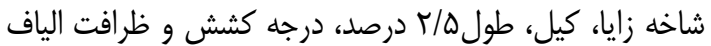

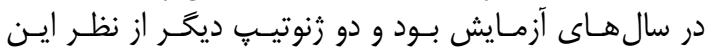

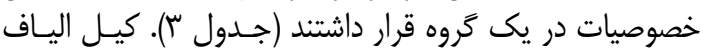

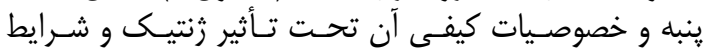

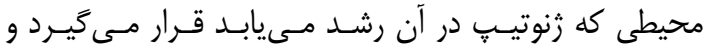

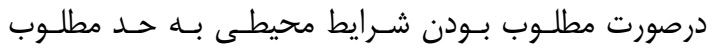

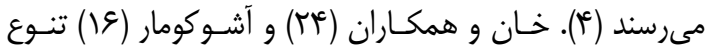

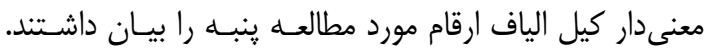

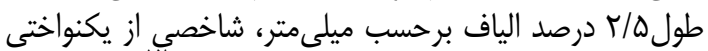

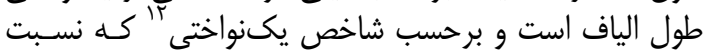

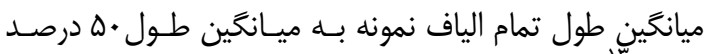

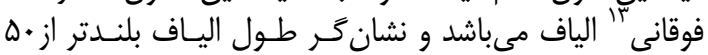

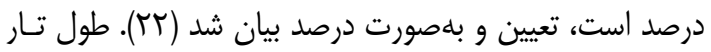

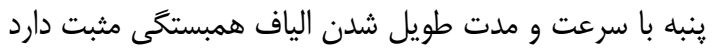

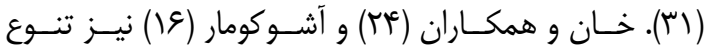

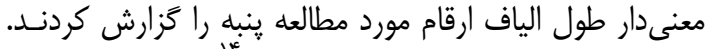

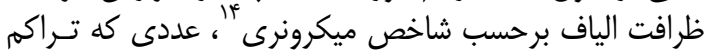

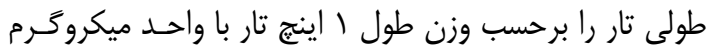

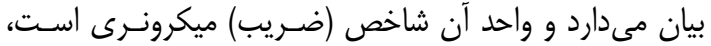

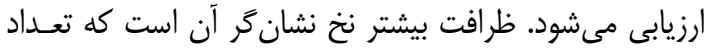

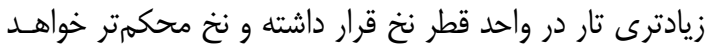

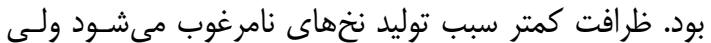

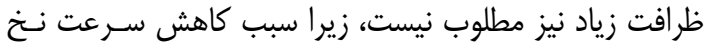

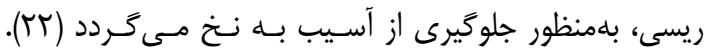

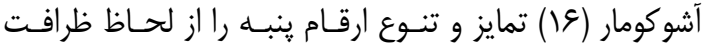

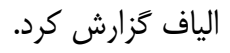

كيل الياف'، از تقسيم وزن الياف به وزن وش هري كر كرت حاصل

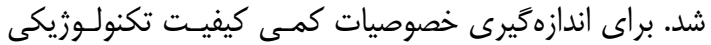

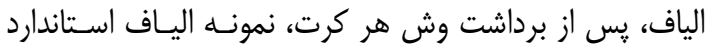

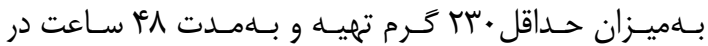

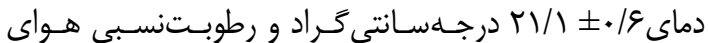

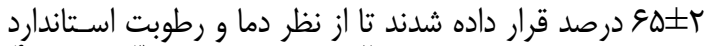

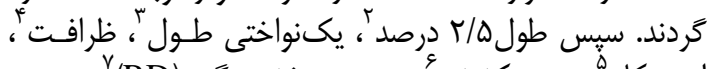

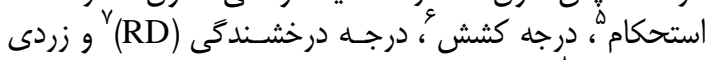

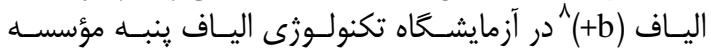

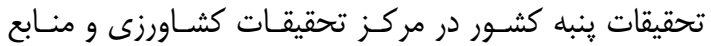

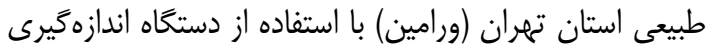

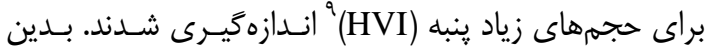

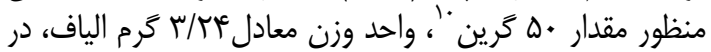

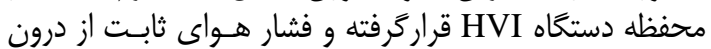

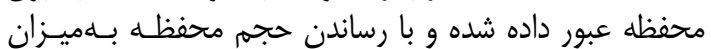

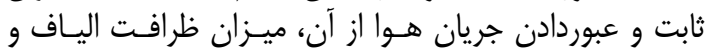

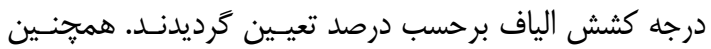

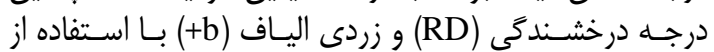
دستخاه HVI اندازمخيرى شدند.

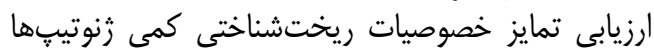

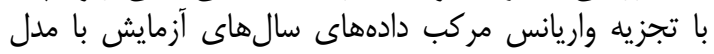

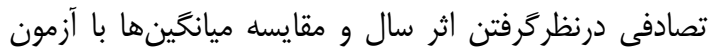

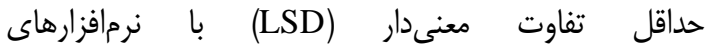
ANAL (ver. 9)

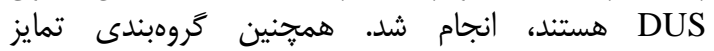
ريختشناختى كيفى ثنوتيبها، بان نرمافزار NTSYS طريق الكوريتم UPGMA انجام شد.

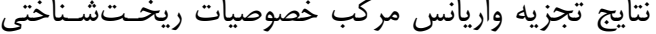

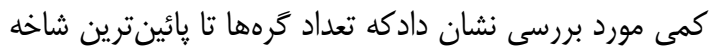

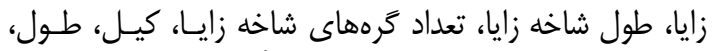

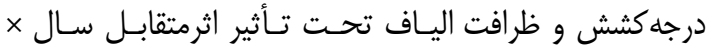

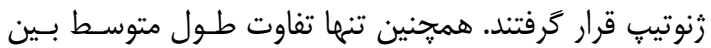

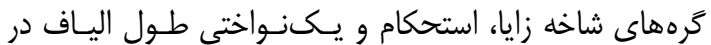

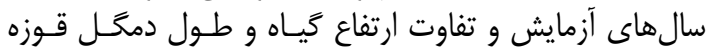

\begin{tabular}{llll}
\hline 1- Ginning outturn & 2- Span length 2.5\% & 3- Fiber length uniformity & 4- Fiber fineness \\
5- Fiber strength & 6- Fiber elongation & 7- Reflectance Degree (RD) & \\
8-Yellowness or Brightness (+b) & 9- High volume Instruments (HVI) & & 10- Grin \\
11- Distinctness, Uniformity and Stability Trial (DUST) & 12- Length uniformity index & 14- Micronaire index \\
13- Upper-half mean length & & 14-
\end{tabular}

13- Upper-half mean length

14- Micronaire index 
جدول r- تجزيه واريانس مركب خصوصيات ريختشناختى كمى مورد ارزيابى زنوتيٍهاى جديد پֶنبه در سالهاى (q-•وس| Table 2. Combined analysis of variance of quantitative morphological traits of evaluated new cotton cultivars during 2011-12 years

\begin{tabular}{|c|c|c|c|c|c|c|c|c|c|c|c|c|c|}
\hline \multicolumn{13}{|c|}{ ميانكَين مربعات } & \multirow[b]{2}{*}{ تغييرات } \\
\hline يكنواختى & ظرافت & كشش الياف & استحكام & 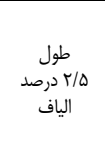 & كريل & قول & كارتفاع & 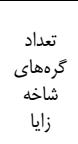 & شاخه & طول متوسط كرهاى & 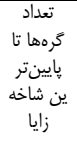 & آزادى دره & \\
\hline$r \mathrm{rWV} / . \mathrm{gr}^{* * *}$ & $V N / T T V^{* *}$ & $r \mid f / W r^{* *}$ & $\Delta V / V / \Lambda q q^{* *}$ & $\Delta T r g / N r^{* *}$ &.$/ M f^{\mathrm{ns}}$ & $\cdot / \Lambda \cdot \gamma^{*}$ & /.r. ns & $1 / \& \Delta \cdot{ }^{\mathrm{ns}}$ & $r / \cdot \wedge f^{* * *}$ & $\cdot / \cdot 10^{\mathrm{ns}}$ & $\cdot / \cdot r^{\mathrm{ns}}$ & 1 & سال \\
\hline$\cdot / \cdot \wedge \Delta$ &.$/ .1 f$ & rו./. & . INES & - IMAF & $.1 .9 V$ & $.1 \cdot \mathrm{nf}^{\mathrm{f}}$ & 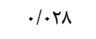 & T4א/ & ./1rq & $\cdot / \cdot 1 F$ &.$/ 111$ & r & تكرار (سال) \\
\hline$\cdot / \cdot v r^{\mathrm{ns}}$ & $.1 .91^{* *}$ & $\cdot / V^{* * *}$ & $1 / . r^{n s}$ & $r M / I V q^{* * *}$ & glgs & $\varphi / \wedge 1 \Delta^{* *}$ & $\mathrm{TV} / \Delta F D^{* *}$ & 1/q4.** &.$/ 911^{*}$ & nirins & $1 / \mathrm{V} \cdot{ }^{* *}$ & r & رنوتيب \\
\hline$\cdot / \cdot v \cdot{ }^{\mathrm{ns}}$ & $. / .9 .^{* *}$ & $\cdot / / v \cdot^{* *}$ & $\cdot(\cdot)^{\mathrm{ns}}$ & $r \cdot / . .{ }^{* *}$ & . $/ \omega^{n s}$ & $\cdot / 1 Q \Delta^{\mathrm{ns}}$ & $\cdot / 190^{\mathrm{ns}}$ & $\cdot \mid 110^{*}$ & ./999* & $\cdot / r F q^{n s}$ & $1 / \Delta V .^{* *}$ & r & سال ×زنوتي \\
\hline.$/ . \Delta \Delta$ & $.1 .1 f$ & .1 .11 &.$/ T F$. & . Ivef & .1 .94 & $.1 \cdot \wedge \mathrm{V}$ & $.1 . v 9$ &.$/ r$ & . & .1 .91 &.$/ N T$ & 8 & آزمايش اشتباه \\
\hline$\cdot / \Delta F$ & $F / T r$ & $r / \Delta q$ & $\Gamma / \Delta \Delta$ & $\Delta / F T$ & $F / \Delta F$ & $F / \Lambda$. & $\Delta / \mathrm{WV}$ & $V / T \Delta$ & $V / q r$ & $11 / 8$ & S/M & & ضغريبرات \\
\hline
\end{tabular}

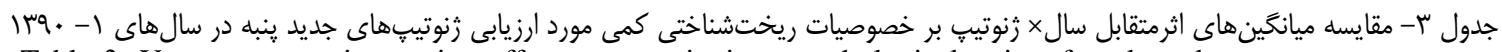

Table 3. Yearxgenotype interaction effect on quantitative morphological traits of evaluated new cotton genotypes mean comparisons during 2011-12 years

\begin{tabular}{|c|c|c|c|c|c|c|c|}
\hline & & شصوصيات ريخت & & & & & \\
\hline 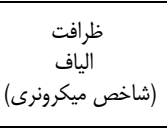 & كشش الياف & $\begin{array}{c}\text { (م/ درصد الياف } \\
\text { (ميلىمتر) }\end{array}$ & شاخر تهاى & 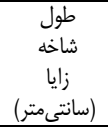 & بإيينترين شاخه زايا & زنوتيب & سال \\
\hline$\Gamma / \Lambda \Delta^{b}$ & $V / F^{b}{ }^{b}$ & $r \Delta / \Lambda \cdot^{b}$ & $r / 4 \cdot{ }^{b}$ & $94 / 1 .{ }^{b}$ & $r / r \Lambda^{b *}$ & GT40 & $1($ (1rq.) \\
\hline$r / u^{b}$ & $V / \Delta \cdot{ }^{b}$ & $r \Delta / q^{\circ}$ & $r / \epsilon^{b} b^{b}$ & $98 / . .^{\mathrm{a}}$ & $F / V^{a}$ & TBL60 & (1) (1\%.) \\
\hline$r / 1 Q^{a}$ & $N / \omega \cdot{ }^{a}$ & $T V / \epsilon^{a}{ }^{a}$ & $r / \Delta \cdot a^{a}$ & $s q / \Delta \cdot^{\mathrm{a}}$ & $r / v^{a}$ & SKT134 & (1) (1\%q.) \\
\hline$r / r^{b}$ & $v / r^{\mathrm{b}}$ & $r \Delta / \Lambda .^{b}$ & $r / r \omega^{b}$ & $9 \Delta / .^{b}$ & $r / T \omega^{b}$ & GT40 & $r(\mid r q))$ \\
\hline$r / q .{ }^{b}$ & $V / g .{ }^{b}$ & $r \& / . .^{b}$ & 每 & $\varepsilon_{\Delta} / q^{a}{ }^{\mathrm{a}}$ & $r / v r^{a}$ & TBL60 & $r(\mid r q))$ \\
\hline$r / .^{a}$ & $N / r^{a}{ }^{a}$ & $r V / . .^{a}$ & $\kappa / r^{\mathrm{a}}$ & $\operatorname{se/r.{}^{\mathrm {a}}}$ & $\kappa / N e^{c^{a}}$ & SKT134 & $r($ (1rq) \\
\hline.$/ r \wedge 9$ & $\cdot|r \Delta|$ & $r / 111$ &.$/ 9 \vee \Delta$ & .1991 & $\cdot|\Lambda|$. & & (هدرصد) LSD \\
\hline
\end{tabular}

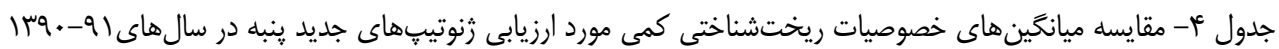
Table 4. Quantitative morphological traits of evaluated new cotton genotypes mean comparisons during 2011-12 years

\begin{tabular}{|c|c|c|c|c|c|c|}
\hline \multirow[b]{2}{*}{ طول "اليفوافتى (درصد) } & \multicolumn{3}{|c|}{ خصوصيات ريختشناختى كمى } & \multirow[b]{2}{*}{ 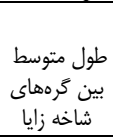 } & \multirow[b]{2}{*}{ 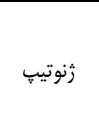 } & \multirow[b]{2}{*}{ سال س } \\
\hline & الياف (كرم بر متحر طول تار) & طول دمكل قوزه & 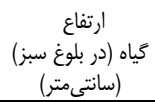 & & & \\
\hline$M / \Delta \cdot{ }^{a}$ & $r \in / q .^{a}$ & $s / \Delta \Delta^{a}$ & $119 / 1 \cdot^{a}$ & $11 / .{ }^{a} *$ & & 1(1\%q.) \\
\hline \multirow[t]{4}{*}{$\Delta \kappa / q .{ }^{b}$} & $r r / 9 .{ }^{b}$ & $\Delta / \cdot \Delta^{\mathrm{b}}$ & $\| \varepsilon / r^{b}$ & $1.1 . .^{b}$ & & $r($ (ाॅ१) \\
\hline & & $s /\left.\Delta\right|^{a}$ & $11 \omega / \Gamma q^{c}$ & & GT40 & \\
\hline & & $9 / 99^{\mathrm{a}}$ & $119 / 9 r^{a}$ & & TBL60 & \\
\hline & & $\Delta / r \omega^{b}$ & $\| V / a r^{b}$ & & SKT134 & \\
\hline
\end{tabular}

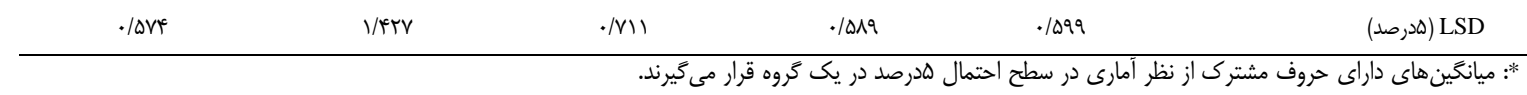

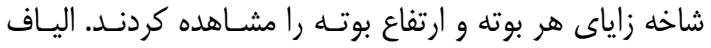

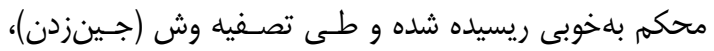

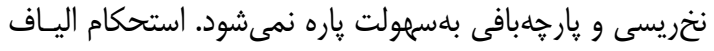

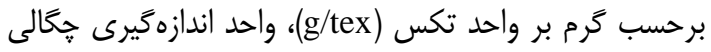

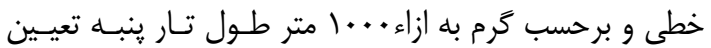

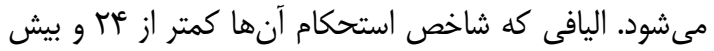

طول متوسط بين گرههاى شاخه زايا، ارتفاع گيـاه، طـول

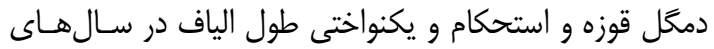

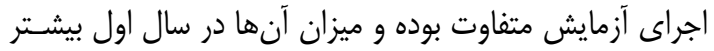

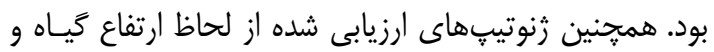

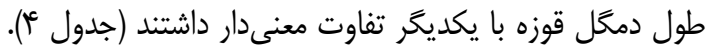

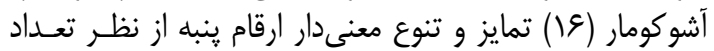




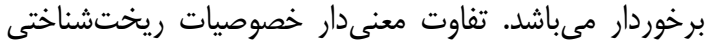

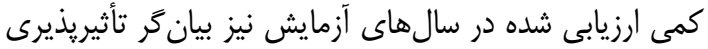

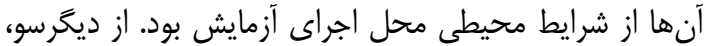

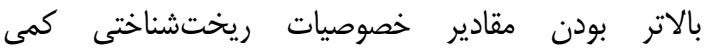

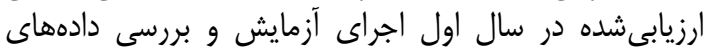

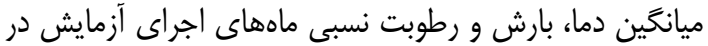

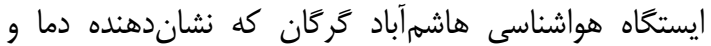

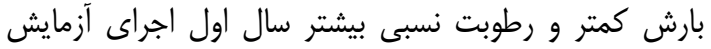

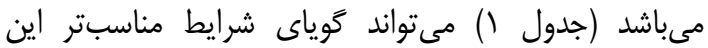

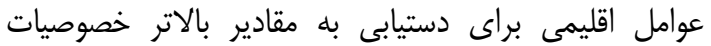

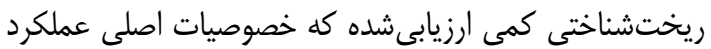

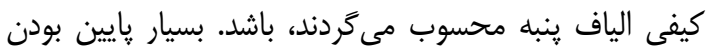

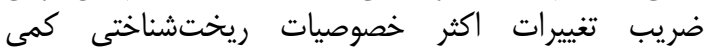
ارزيابىشده مىتواند بيانكر يكنواختى مشاهده شدهات اين اين

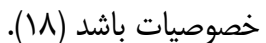
خصوصيات ريختشناختى كيفى

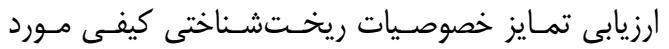

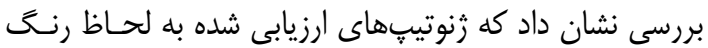

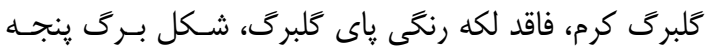

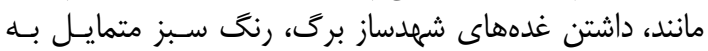

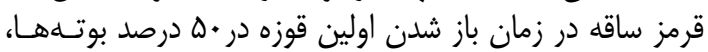

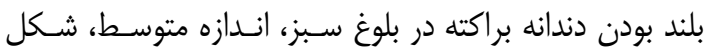

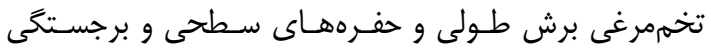

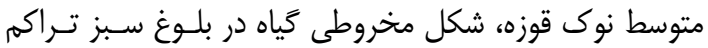

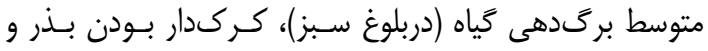

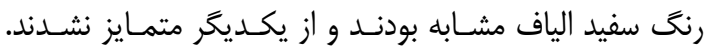

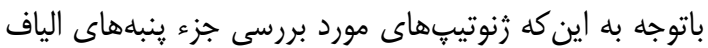

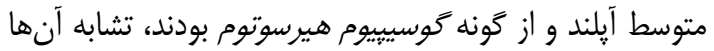

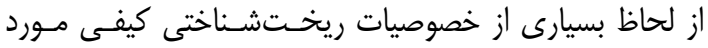

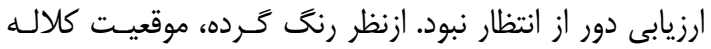

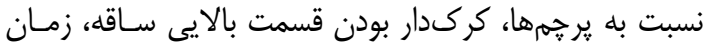

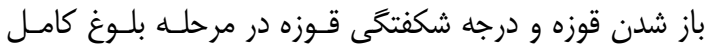

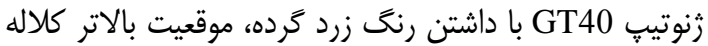

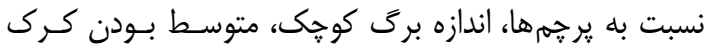

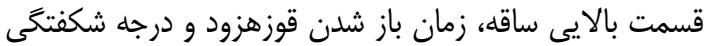

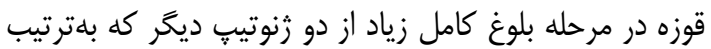

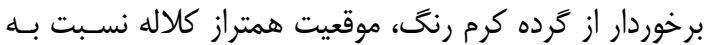

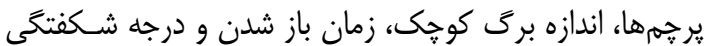

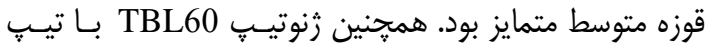

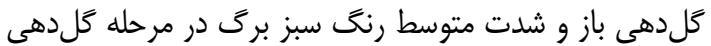

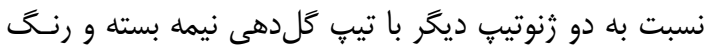

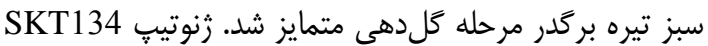

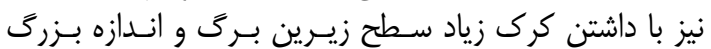

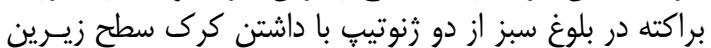

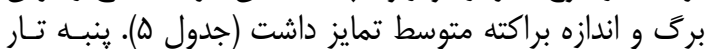

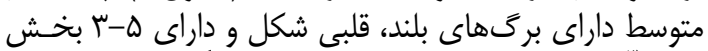

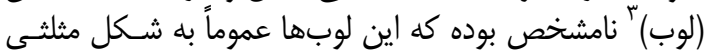

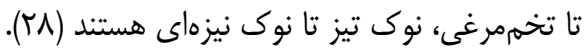

از • • طرم بر تكس (g/tex) است بلترتيب الياف با استحكام

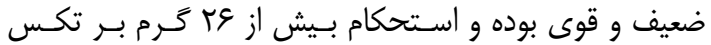
(g/tex)

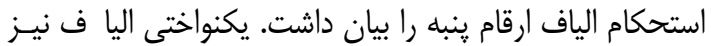

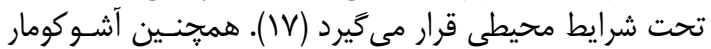

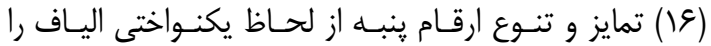

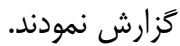

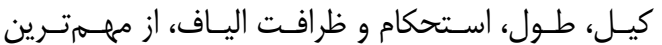

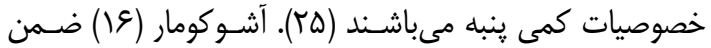

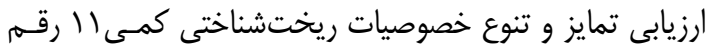

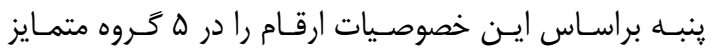

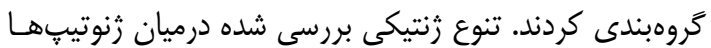

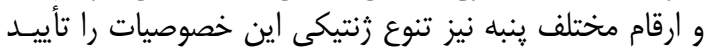

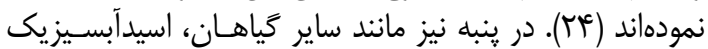

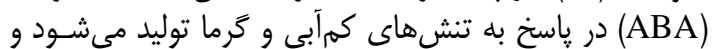

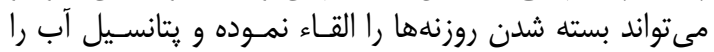

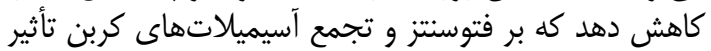

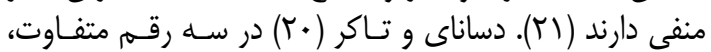

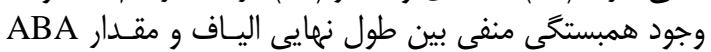
را كزارش نمودند. كاهش سرعت فتوسنتز و ساير عوامل متابوليكى كه شدت

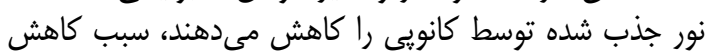

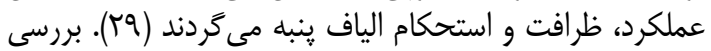

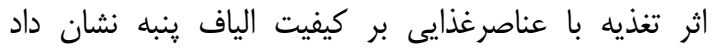

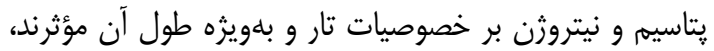

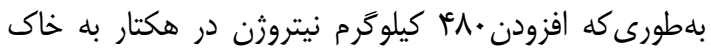

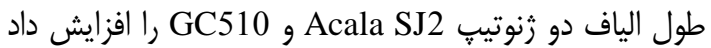

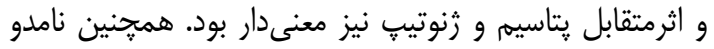

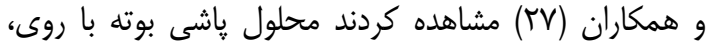

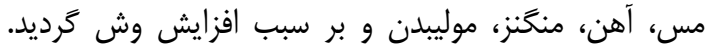

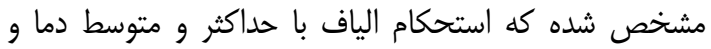

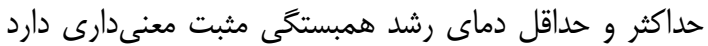

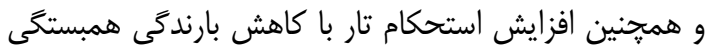

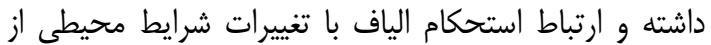

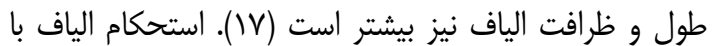

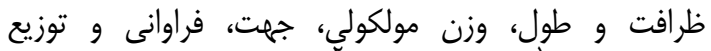

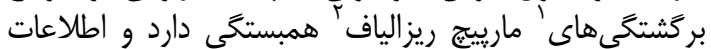

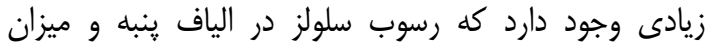

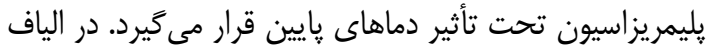

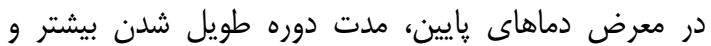

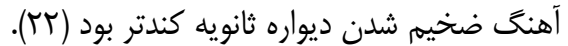

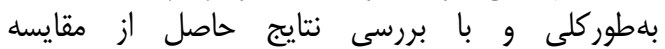

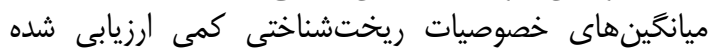

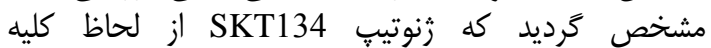

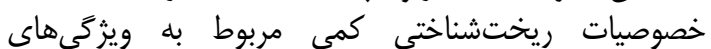

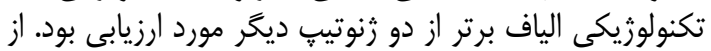

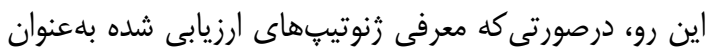

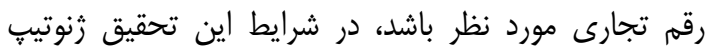
SKT134 از شايستخى بيشترى براى معرفى بهاعنوان رقهم

4- Random Amplified Polymorphic DNA (RAPD) 


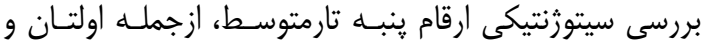

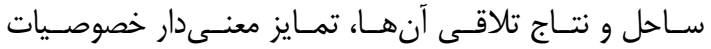

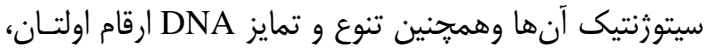
بختخان و ساحل و نتاج تلاقى آنها را كزارش نمودند.

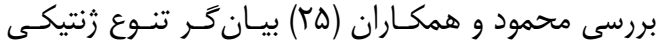

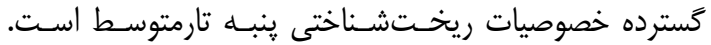

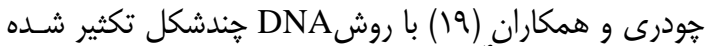

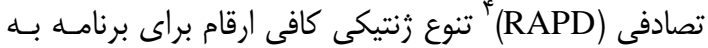

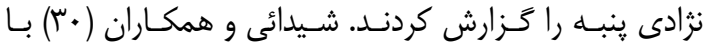

جدول ه- تظاهر خصوصيات ريختشناختى كيفى زنوتيڤهاى جديد ينبه مورد ارزيابى Table 5. Qualitative morphological traits expression of evaluated new cotton genotypes

\begin{tabular}{|c|c|c|c|c|c|c|c|c|c|c|}
\hline \multicolumn{10}{|c|}{ خصوصيات ريختشناختى كيفى } & \multirow[b]{2}{*}{ رُنوتيب } \\
\hline 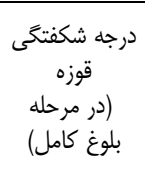 & 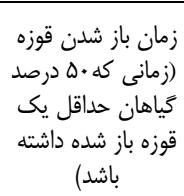 & قبالايى & 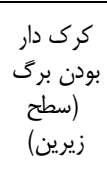 & برى اندازه & 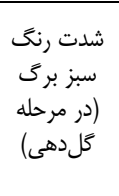 & 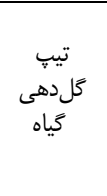 & 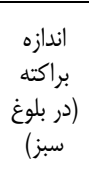 & 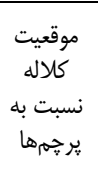 & رَرده & \\
\hline زياد & زود & متوسط & متوسط & كوجى & تيره & نيمه بسته & متوسط & بالاتر & زرد & GT40 \\
\hline متوسط & متوسط & زياد & متوسط & متوسط & متوسط & باز & متوسط & همتراز & كرم & TBL60 \\
\hline متوسط & متوسط & زياد & زياد & متوسط & تيره & نيمه بسته & بزرى & همتراز & كرم & SKT134 \\
\hline
\end{tabular}

وجود ميزان اين خصوصيات ريختشناختى كمى در سال إناى

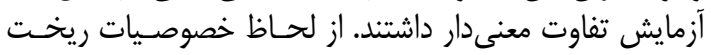

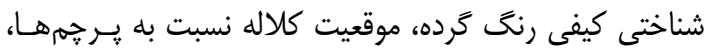

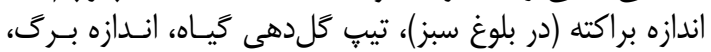

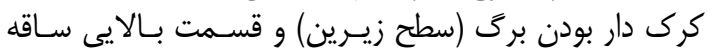

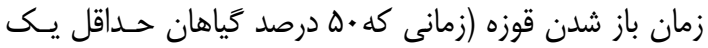

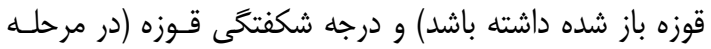

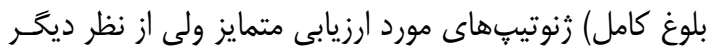

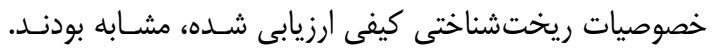

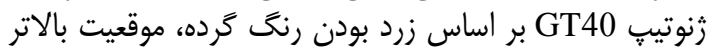

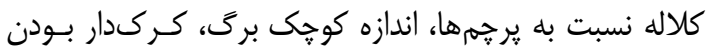

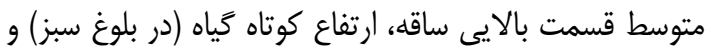

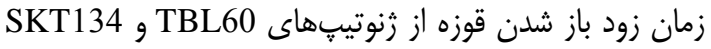

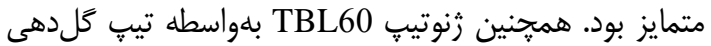

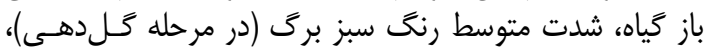

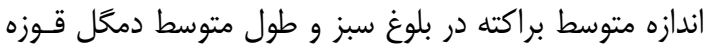

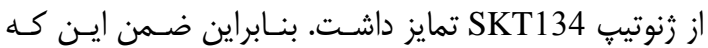

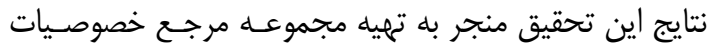

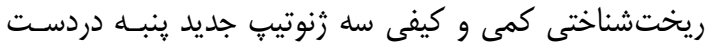

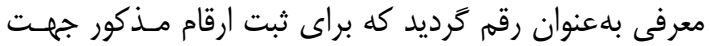

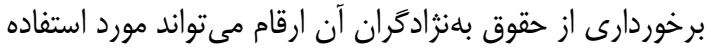

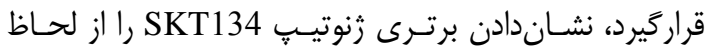

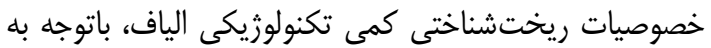

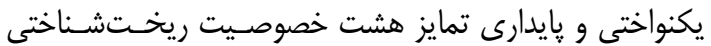

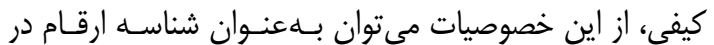

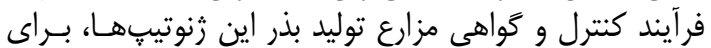
ارزيابى خلوص و اصالت زنتيكى، استفاده نمود.
بهمنظور از درجه رنغ كه براى ارزيابى رنغ الياف استفاده

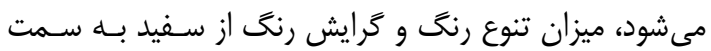

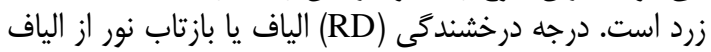

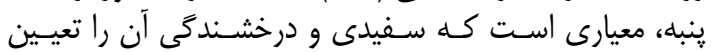

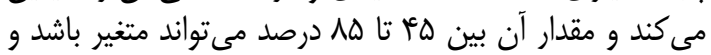

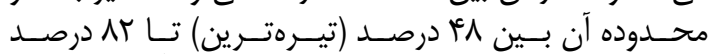

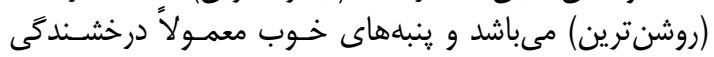

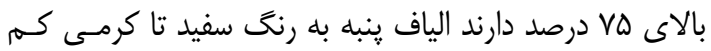

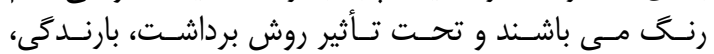

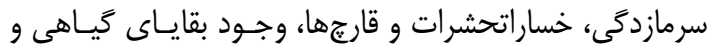

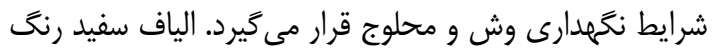

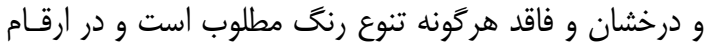

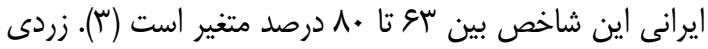

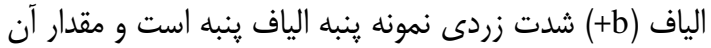

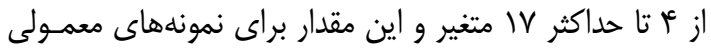

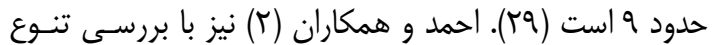

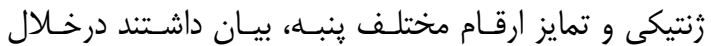

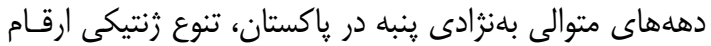
جديد ينبه رو به كاهش كذاشته بله است.

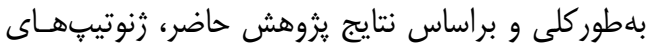

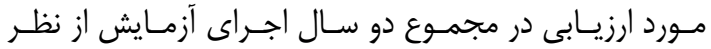

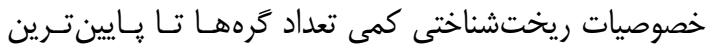

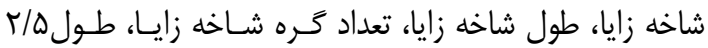

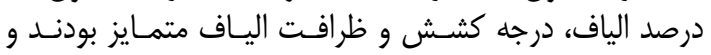

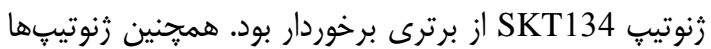

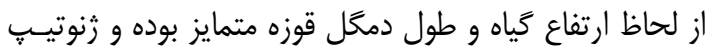

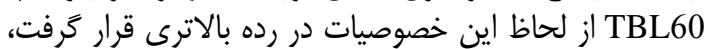

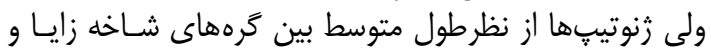

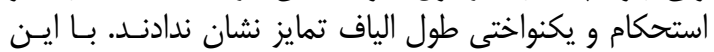


1. Agrawal, P.K. 2002. Cultivar purity test, In: Agrawal, P.K. (ed.) Principles of seed technology. Indian Council of Agricultural Research, New Delhi, 96-104 pp.

2. Ahmad, M.Q., S.H. Khan and F.M. Azhar. 2012. Decreasing level of genetic diversity in germplasm and cultivars of upland cotton (Gossypium hirsutum) in Pakistan. Journal of Agricultural and Social Science, 8: 92-96.

3. Alishah, O. 2007. Special words of cotton. Ministry of Jihad-e-Agriculture, Agricultural Research Education and Extension Organization (AREEO), Extension and Education Deputy, Agriculture Education Publication, $268 \mathrm{pp}$ (In Persian).

4. Allen, R.D. and L. Aleman. 2011. Abiotic stress and cotton fiber development. In: Oosterhuis D.M. (ed.) Stress physiology in cotton. Number Seven The Cotton Foundation Reference Book Series, The Cotton Foundation Cordova, Tennessee, U.S.A, 150-160 pp.

5. Anonymous. 2015. OECD schemes for the varietal certification or the control of seed moving in international trade, annex I, OECD control plot and field inspection. Organization for Economic Cooperation and Development (OECD). Paris.

6. Anonymous. 2001. Guidelines for the conduct of tests for distinctness, uniformity and stability Cotton (Gossypium L.). International Union for the Protection of New Varieties of Plants (UPOV). Geneva.

7. Anonymous. 2007. National guidelines for the conduct of tests for distinctness, uniformity and stability in tetraploid cotton. Ministry of Jihad-e-Agriculture, Agricultural Research Education and Extensions Organization (AREEO), Seed and Plant Certification and Registration Institute (SPCRI), (In Persian).

8. Anonymous. 2011. Golestan province weather 2011almanac. Golestan province meteorology office scientific gazette (In Persian).

9. Anonymous. 2011. OECD schemes for the varietal certification of crucifer seed and other oil or fiber species seed, moving in international trade. Annex VII to the decision. Organization for Economic Co-operation and Development (OECD). Paris.

10. Anonymous. 2012. Iran plant varieties national list ( $1^{\text {st }}$. vol. Agricultural crops).Ministry of Jihad-eAgriculture, Agricultural Research Education and Extensions Organization (AREEO), Seed and Plant Certification and Registration Institute (SPCRI), (In Persian).

11. Anonymous. 2012. Golestan province weather 2012almanac. Golestan province meteorology office scientific gazette (In Persian).

12. Anonymous. 2014. Cotton world supply, use, and trade. Available at www.fas. usda.gov/cotton /current.

13. Anonymous. 2014. Long term Golestan province weather almanac. Golestan province meteorology office scientific gazette.

14. Anonymous. 2017. Agriculture statistics, first volume-horticultural and field crops, 2014-15 crop year. Information and Communication Technology Center of Ministry of Jihad-e-Agriculture.

15. Anonymous. 2017. OECD seed schemes 2017, OECD schemes for the varietal certification or the control of seed moving in international trade. Organization for Economic Co-operation and Development (OECD). Paris.

16. Ashokkumar, K. 2011. Morphological Diversity and per se Performance in Upland Cotton (Gossypiumhirsutum L.). Journal of Agricultural Science, 3(2): 107-113.

17. Bradow, J.M. and G.H. Davidonis. 2010. Effect of environment on fiber quality. In: Stewart, J. McD., D. Oosterhuis, J.J. Heitholt and J. Mauney. (eds.) Physiology of Cotton, Springer Science+Business Media B.V, 229-245 pp.

18. Bowman, D.T. 2001. Common use of the CV: a statistical aberration in crop Performance trials. The Journal of Cotton Science, 5: 137-141.

19. Chaudhary, L., A. Sindhu, M. Kumar, R. Kumar and M. Saini. 2010. Estimation of genetic divergence among some cotton varieties by RAPD analysis. J. Plant Breeding Crop Science, Vol, 2(3): 039-043.

20. Dasani, S.H. and V.S. Thaker. 2006. Role of abscisic acid in cotton fiber development. Russian Journal of Plant Physiology, 53: 62-67.

21. Finkelstein, R.R., S.S. Gampala and C.D. Rock. 2002. Abscisic acid signaling in seeds and seedlings. Plant and Cell, 14: 15-45.

22. Haigler, C.H. 2010. Physiological and anatomical factors determining fiber structure and utility. In: Physiology of Cotton, By: Stewart, J.McD., Oosterhuis, D., Heitholt, J.J. and Mauney, J. (eds.), Springer Science+Business Media B.V, 33-47 pp.

23. Hamidi, A., K. Ghasemi Bazdi, E. Baniani, M.H. Hekmat, O. Alishah, M. Arab Salmani, M.R. Vafai Tabar, A.A. Miri and F. Khazae. 2016. Evaluation of distinctness, uniformity and stability of Cotton (Gossypium hirsutum L. and G. barbadense) common and new cultivars by using morphological characteristics. Iranian Journal of Cotton Researches, 3(2): 1-25.

24. Khan, A.I., F.S. Awan, B. Sadia, R.M. Rana and I.A. Khan. 2010. Genetic diversity studies among coloured cotton genotypes by using RAPD markers. Pakistan Journal of Botany, 42(1): 71-77.

25. Mahmood, S., M. Irfan, F. Raheel and A. Hussaim. 2006. Characterization of cotton (Gossypium hirsutum L.) varieties growth and productivity traits under water deficit conditions. International Journal of Agriculture and Biology, 8(6): 796-800.

26. Mozafari, J., S.Y. Sadeghian, S. Mobasser, H. Khademi and S.A. Mohammadi. 2010. Principles of plant variety protection. Ministry of Jihad-e-Agriculture Agricultural Research Education and Extensions Organization (AREEO), Seed and Plant Certification and Registration Institute (SPCRI), $446 \mathrm{pp}$ (In Persian).

27. Namdeo, K.N., J.K. Sharma and K.C. Mandloi. 1992. Effect of foliar feeding of micronutrients on production of rainfed hybrid cotton. Crop Research hisar, 5(3): 451-455. 
$V^{m}$

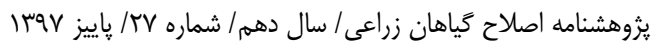

28. Nikolić, Z., M. Vujakovic and A. Jevtic. 2008. Genetic purity of sunflower hybrids determined on the basis of isozymes and seed storage proteins. Helia, 31(48): 47-54 pp.

29. Pettigrew, W.T., J.J. Heitholt and W.R. Meredith. 1996. Genotypic interactions with potassium and nitrogen in cotton of varied maturity. Agronomy Journal, 88: 89-93.

30. Sheidai, M., A. Golestanipoor and E. Jorjani. 2006. Chromosome pairing and heterozygote translocation in oltan cotton cultivar and its crossing progenies. Iranian Journal of Science and Technology Transaction, 30: 103-108.

31. Sheidai, M., A. Dokhanchei, Z.H. Shahriari, Z. Noormohammadi and F. Farahanei. 2007. Study of genetic polymorphism in some tetraploid cotton cultivars by using RAPD analysis. Pakistan Journal of Biological Science, 10(16): 2748-2751.

32. Tianzhen, Z. and P. Jiaju. 2000. Hybrid seed production in cotton. In: Basra, A.S. (ed.), Heterosis and hybrid seed poroduction in agronomic crops. Food Products Press, 149-184 pp.

33. Umarani, R., R. Jerlin, N. Natarajan, P. Masilmani and A.S. Ponnuswamy. 2006. Cultivar identification, In: Experimental seed science and technology, Agrobios, India, 93-101 pp. 


\title{
Evaluation of Morphological Characteristics of Cotton (Gossypium hirsutum L.) New Genotypes in Golestan Province
}

\author{
Aidin Hamidi ${ }^{1}$, Kamal Ghasemi Bazdi ${ }^{2}$ and Yasser Jafari ${ }^{3}$
}

1- Associate Professor, Agriculture Research, Education and Extension Organization (AREEO), Seed and Plant Certification and Registration Institute (SPCRI), (Corresponding author: a.hamidi@areeo.ac.ir)

2 and 3- Associate Professor and Expert, Agriculture Research, Education and Extension Organization (AREEO), Cotton Research Institute (CRI)

Received: July 7, 2015

Accepted: August 18, 2018

\begin{abstract}
In order to evaluate of distinctness. uniformitv and stabilitv (DUS) of morphological traits, three new genotypes of cotton (GT40, TBL60 and SKT134) were tested using a complete randomized blocks design with 4 replications. In general, 39 quantitative and qualitative morphological characteristics were measured. Results showed quantitative morphological characteristics including number of stem nodes to the lowest fruiting branch (at flowering stage), fruiting branch length, fruiting branch nodes number, ginning outturn, fiber length, elongation and fineness, plant height and boll petiole length of genotypes significantly were distinct. Also genotypes qualitative morphological characteristics: pollen color, position of stigma relative to anthers, bract size (at green maturity), type of flowering, intensity of leaf green color (at flowering stage), Leaf size and pubescence of lower side, stem pubescence in upper part, boll opening time (when 50\% of the plants have at least one boll opened) and degree of opening (at full maturity) significantly were distinct. Generally, achieved results showed, GT40 genotype based on yellow pollen color, upper position of stigma relative to anthers, small leaf size, medium stem pubescence in upper part, short plant height (at green maturity) and early boll time of opening (when $50 \%$ of the plants have at least one boll opened) was distinct from TBL60 and SKT134 genotypes. Also TBL60 genotype due to non-clustered type of flowering, medium leaf intensity of green color (at flowering stage), medium bract size (at green maturity) and medium boll petiole length were distinct from SKT134 genotype. Therefore, this research results characterized distinct morphological characters having uniformity and stability of three cotton new genotypes in the course of introduction as cultivars which could be used for mentioned cultivars registration for having plants breeders' right. Also, with due attention to uniformity and stability of distinctness of eight qualitative morphological characteristics, can used these characteristics as descriptors of cultivars which would introduced for those genotypes seed production fields control and certification of genetical purity and genuineness.
\end{abstract}

Kevwords: Cotton. Cultivar registration, Distinctness Uniformity, Stability, Morphological characteristic 